\title{
Improving Smoking Cessation After Myocardial Infarction by Systematically Implementing Evidence-Based Treatment Methods: A Retrospective Observational Cohort Study
}

\section{Margret Leosdottir ( $\square$ Margret.Leosdottir@med.lu.se ) \\ Lund University}

\section{Sanne Wärjerstam}

Skåne University Hospital

Halldora Michelsen

Helsingborgs lasarett

Mona Schlyter

Skåne University Hospital

Emma Hag

Ryhov Hospital Jönköping

John Wallert

Karolinska Institute

\section{Matz Larsson}

Örebro University Hospital

\section{Research Article}

Keywords: Smoking cessation, myocardial infarction, cardiac rehabilitation, varenicline, nicotine replacement therapy, implementation.

Posted Date: September 27th, 2021

DOI: https://doi.org/10.21203/rs.3.rs-823380/v2

License: (c) (1) This work is licensed under a Creative Commons Attribution 4.0 International License. Read Full License

Version of Record: A version of this preprint was published at European Heart Journal on October 1st, 2021. See the published version at https://doi.org/10.1093/eurheartj/ehab724.1409. 


\section{Abstract}

We compared the odds of smoking cessation at 2-months post-myocardial infarction (MI) before and after implementing routines, optimizing the use of evidence-based methods for smoking cessation, with start during admission. The following routines were implemented at six hospitals in Sweden: cardiac rehabilitation nurses offering smokers short consultation during admission, optimizing nicotine replacement therapy, increasing prescription of varenicline, and contacting patients by telephone during the 1st week post-discharge. Using logistic regression, odds for smoking cessation at 2-months post-MI before $(n=188)$ and after $(n=195)$ routine implementation were compared. Secondary outcomes included adherence to implemented routines. After implementation, a larger proportion of patients (65\% vs $54 \%$ ) were abstinent at 2-months (OR 1.60 [1.04-2.48], p = 0.034). Including only those counselled during admission, 74\% were abstinent (OR 2.50 [1.42-4.41], $p=0.002$ ). After implementation patients were more often counselled during admission ( $50 \%$ vs $6 \%, p<0.001)$, prescribed varenicline $(23 \%$ vs $7 \%$, $p<0.001)$, and contacted by telephone post-discharge ( $18 \%$ vs $2 \%, p<0.001)$. Being prescribed varenicline had the strongest association with smoking abstinence at 2-months (adjusted OR 4.09 [1.6810.00], $p=0.002$ ). In conclusion, readily available methods for aiding smoking cessation can be implemented effectively in routine practice, with beneficial effects on smoking cessation for the high-risk group of smoking MI patients.

\section{Introduction}

Smoking is one of the primary risk factors for coronary heart disease (CHD) ${ }^{1}$. For smokers who suffer a myocardial infarction (MI), ceasing to smoke after the $\mathrm{MI}$ is the most effective of all preventive measures. Similarly, tobacco abstinence is associated with a lowered risk of reinfarction by $30-40 \% 2,3$ and death by $45 \%{ }^{4}$. Supporting patients to quit smoking after an $\mathrm{Ml}$, and to remain abstinent long-term, are therefore primary goals of cardiac rehabilitation (CR).

The Swedish quality registry SWEDEHEART (The Swedish Web-system for Enhancement and Development of Evidence-based care in Heart disease Evaluated According to Recommended Therapies) registers data from an unselected MI-patient population, with nationwide coverage and one-year followup data available for $>80 \%$ of those $<80$ years of age (www.swedeheart.se). According to the most recent SWEDEHEART annual report, the proportion of active smokers among patients with MI in Sweden was $23 \%$, which also has decreased successively over the last decade alongside a general population reduction in daily smoking ${ }^{5,6}$. The proportion of smokers who reported being abstinent at 2-months postMI was $57 \%$ and $56 \%$ at one-year post-Ml, which is somewhat higher than the European average of $~ 45 \%$ 6,7 . However, no visible progress in smoking abstinence rates among Swedish patients with $\mathrm{MI}$ has been observed for more than ten years (Fig. 1) ${ }^{6}$.

Current CR guidelines advocate professional support, with smoking cessation programmes initiated already during hospital admission ${ }^{8,9}$. Drug interventions are also strongly recommended, using nicotine 
replacement therapy (NRT), varenicline and/or bupropion ${ }^{8,9}$. Varenicline is the most effective medical treatment to support smoking cessation ${ }^{10}$. Recent studies have confirmed its safety not only in patients with stable $\mathrm{CHD}$ but also in patients with acute coronary syndromes, including $\mathrm{MI}^{11-13}$. In the fifth and most recent European Action on Secondary and Primary Prevention by Intervention to Reduce Events (EUROASPIRE) survey, most currently smoking patients with MI were offered personal advice by a healthcare professional to stop smoking but NRT was prescribed to only $7 \%$ of smokers and varenicline to $2 \%$, with no meaningful change between EUROASPIRE III and V 7,14. Consequently, there is clearly a considerable room for improvement with respect to medical treatment for active smokers with $\mathrm{Ml}$.

\section{Methods}

The aim of this study was to compare the odds of smoking cessation at 2-months post-MI before versus after implementing a set of pre-specified routines for optimization of evidence-based treatment methods for smoking cessation, with start during admission.

\section{Study design}

A retrospective observational multicentre cohort study.

\section{Setting and participants}

As a joint venture for clinical quality improvement, structured routines for early smoking cessation counselling and treatment optimization were implemented at six hospitals in Sweden in 2018-2019. Two are university hospitals. The largest hospital is situated in Malmö, the third largest city in Sweden. Two are of medium size (Örebro and Jönköping) and three are smaller urban or rural hospitals (Trelleborg, Eksjö and Värnamo). The three largest hospitals have acute percutaneous coronary intervention facility, and all have a coronary intensive care unit and an outpatient cardiac rehabilitation (CR) centre. The CR team in Malmö was the first to start implementing the new working routines. The Malmö team thereafter visited the other hospitals to introduce the $\mathrm{CR}$ and coronary intensive care personnel to the new routines and support implementation.

The participating CR centres report to the SWEDEHEART registry ${ }^{6}$ and provide comprehensive CR to postMI patients. The "usual care" CR programme starts with an individual patient assessment with a nurse at 2-3 weeks after discharge, followed by two registry-based nurse visits at 6-10 weeks (2-month visit) and 11-13 months (1-year visit). Central components of the programme include control and treatment of cardiovascular risk factors, interactive patient education, psychosocial management, smoking cessation counselling (individual or group therapy) if needed, individual patient assessment with a physiotherapist, and supervised centre-based exercise training.

\section{The new routines}

The newly adapted routines included the following: 
- A CR nurse should identify all actively smoking MI-patients while still hospitalized for their MI and offer a short consultation with the patient during admission. A new information pamphlet for patients was designed, which included information on the benefits of smoking cessation, tips for avoiding relapse after discharge, plan for follow-up and contact information for the CR centre and generally available anti-smoking aid (telephone and online support).

- The CR nurse should give advice to the coronary intensive care nurses and physicians on optimal dosage of NRT during admission and encourage prescription at discharge (even though NRT is available over the counter in Sweden), as an attempt to increase adherence.

- The CR nurse should identify eligible patients for treatment with varenicline, and alert the responsible physician, who in turn would initiate treatment if deemed suitable.

- Varenicline treatment should preferably be started before discharge.

- The patient should be contacted by telephone, by the same nurse that consulted the patient during admission, during the first week after discharge. Thereafter, usual care CR commenced.

- If the patient opted out from varenicline treatment during admission and was still smoking at the telephone contact or $1^{\text {st }} \mathrm{CR}$ follow-up visits, treatment should be offered again.

- Finally, centres were encouraged to strive for continuity in nurse-patient care, meaning the same CR nurse should care for the patient from the first contact during admission throughout CR follow-up.

As such, the CR nurse was the key professional in implementing the new routines. CR nurses at all participating centres had tobacco counselling qualifications prior to the start of the project. If requested, a brief refreshment course and coaching guide was provided by a tobacco counsellor. At some centres, the routines were predominantly new, and at others only smaller amendments or refreshing of routines were needed (see Supplementary Table 1).

\section{Data collection and outcomes}

Smoking cessation at 2-months post-MI for currently smoking patients aged 18-74 years admitted to the coronary intensive care units at the participating hospitals with a type-1 $\mathrm{Ml}$, before (reference period) and after (implementation period) routine implementation, were compared. The implementation and reference periods were of equivalent length at each participating hospital. For the three hospitals with the longest implementation periods (8-14 months), the end of the reference period was set one month prior to start of the implementation period. The remaining three hospitals had shorter implementation periods (4 months), and for which the same period the year prior to the implementation year, was used to minimize seasonal bias. The start and length of the study periods per hospital is listed in Supplementary Table 2. Baseline data and smoking status at 2-months was retrieved from the SWEDEHEART registry. Current smoking was self-reported and defined as daily smoking during the last month. Adherence to the pre-set routines was retrieved from patient journal records at each participating hospital.

According to the original study protocol, primary outcome was smoking abstinence at the 1-year followup visit. Since the Covid-19 pandemic started at the time the last patients included in the implementation 
period had their 2-month follow-up - seriously debilitating planned follow-up routines at all CR centres primary outcome was changed to smoking abstinence at 2-months post-MI. Secondary outcomes included (i) adherence to the newly implemented routines and (ii) prognostic value of each of the new routines for smoking abstinence at 2-months. Continuity in nurse-patient care was defined as $2 / 3$ or more of same nurse-patient contact (physical visits during admission and follow-up and telephone contacts during follow-up).

\section{Availability of data and materials}

The SWEDEHEART registry data that support the findings of this study are available from Uppsala Clinical Research Center (UCR) in Sweden but restrictions apply to the availability of these data, which were used under license for the current study, and so are not publicly available. Third-party data usage is not allowed, irrespective of whether the data contain potentially identifying or sensitive data or not. Instead, given ethical study approval from the Swedish Ethical Review Authority, access to SWEDEHEART data supporting the present findings can be applied for from UCR. Further information can be found on the UCR (www.ucr.uu.se/en/) and Swedish Ethical Review Authority (etikprovningsmyndigheten.se) websites. The study protocol has not been previously published but is available from the corresponding author on reasonable request.

\section{Ethics approval and consent to participate}

The Swedish Ethical Review Authority approved the study (2019-06177). As it involved evaluating outcomes after implementing evidence-based and guideline-recommended clinical routines in daily practice, the need for informed consent was waived. As for SWEDEHEART data, a nurse or physician verbally informs all eligible patients at hospital admission of their registration and opt-out rights. All patients have the right to deny registration and retain the right to be removed from the registry at any time.

\section{Sample size calculations}

In the randomized controlled double-blind Evaluation of Varenicline in Smoking Cessation for Patients Post-Acute Coronary Syndrome (EVITA) trial, patients with acute coronary syndrome and who were smokers at baseline were randomized to receive either varenicline or placebo before discharge ${ }^{11}$. In the intervention arm, $58 \%$ of patients were smoke-free at first follow-up (12 weeks), compared to $36 \%$ in the placebo arm (21.3\% difference, $p<0.0001)$. In the current study, at the CR centres implementing the new routines, $59 \%$ of current smokers at the time of MI were abstinent at the first SWEDEHEART follow-up (610 weeks) in $2017^{15}$. Based on EVITA results, we concluded that a $15 \%$-point change improvement in smoking cessation rates was a reasonable target for the current study. A somewhat lower improvement rate compared to EVITA was expected because the proportion of smokers abstinent at 2-months is already quite high and not all patients were expected to be identified and thus potentially benefitting from the new routines. With a power of $80 \%$ and a two-sided significance level of 0.05 we calculated that we need to include 150 patients in each group. 
Baseline patient characteristics are presented as medians $(q 1, q 3)$ and percentages. Adherence to the implemented routines are described using percentages and comparisons made using chi-square test. The primary and secondary outcomes were analyzed using logistic regression. The primary outcome analysis was first done for all current smokers admitted during the predefined implementation period (intention to treat) and thereafter including only current smokers counselled during admission (per protocol). For the secondary outcomes, each routine's association with smoking cessation at 2-months for patients from the implementation period only were evaluated. Finally, all routines were included as independent variables simultaneously. For all regression analyses, crude models were fitted, whereafter models were adjusted for age, gender, size of center (small, medium, large), length of hospital stay, and whether the patient was discharged during the weekend (Saturday-Sunday), Mondays or during holidays. Mondays were included as patients discharged on Mondays often had returned home before the nurse had a chance to consult the patients.

\section{Results}

Implementation of the new routines started in Malmö in October 2018 with the last hospitals (Eksjö and Värnamo) joining in September 2019 (see Additional file 3). The time frame for patient inclusion ended early January 2020, when 195 current smokers had been admitted for MI at the participating hospitals (Figure 2). During the pre-specified reference period, 188 active smokers were admitted. A somewhat higher number of patients were included in the analysis than indicated by the sample size calculations, to compensate for attrition. Baseline characteristics are shown in Table 1. Median age was 60 (range 33-74) years in both groups. The proportion of men was somewhat higher in the reference group $(77 \% \mathrm{vs} 71 \%$, $\mathrm{p}=0.1)$.

\section{Primary outcome}

In total, 159 out of the 188 (85\%) and 179 out of the 195 (92\%) active smokers at baseline during the reference and implementation periods, respectively, attended a 2-month follow-up. Including all these patients, irrespective of whether they had been subject to any of the new routines or not (intention to treat), significantly more patients were abstinent from smoking at the 2-month follow-up after the new routines had been implemented: $64 \%$ compared to $54 \%$ during the reference period ( $10 \%$ difference, crude OR 1.60 [1.04-2.48], p=0.034; adjusted OR 1.60 [1.03-2.52], p=0.037;) (Figure 3). Comparing only patients who were counselled by a CR nurse during admission ( $50 \%$ of the patients during the implementation period - per protocol), $73 \%$ were abstinent at the 2-month follow-up, compared to $54 \%$ during the reference period (19\% difference, crude OR 2.50 [1.42-4.41], $p=0.002$; adjusted OR 2.68 [1.46-4.91], $\mathrm{p}=0.002$ ).

\section{Secondary outcomes}


During the implementation period, patients were more often counselled during admission ( $50 \% \mathrm{vs} 6 \%$, $p<0.001)$, prescribed varenicline at discharge $(14 \%$ vs $2 \%, p<0.001)$ and during follow-up (up until and including the 2-month follow-up visit: $12 \%$ vs $5 \%, p=0.011$; at discharge or during follow-up combined: $23 \%$ vs $7 \%, p<0.001)$. Patients slightly more often received an NRT prescription at discharge $(21 \%$ vs $14 \%$, $p=0.051$ ) but less often during follow-up ( $5 \%$ vs $12 \%, p=0.017$; at discharge or during follow-up combined: $23 \%$ vs $16 \%, p=0.093)$. During the intervention period, patients were more often contacted by telephone during the first post discharge week, compared to during the reference period ( $18 \%$ vs $2 \%, p<0.001)$. The total number of contacts (by telephone or visits) during the 2-month follow-up period was higher during the intervention period ( 2.8 vs 2.3 contacts, $p<0.001$ ) and more patients had continuity in their nursepatient care ( $64 \%$ vs $51 \%, \mathrm{p}=0.010)$. During the implementation period, comparing patients counselled during admission vs those not counselled, $33 \%$ vs $14 \%$ received a varenicline prescription at discharge or during follow-up ( $\mathrm{p}=0.002), 30 \%$ vs $17 \%$ received a NRT prescription at discharge or during follow-up $(p=0.033)$, and $36 \%$ vs $0 \%$ were contacted by telephone during the first week after discharge $(p<0.001)$. Patients not counselled during admission more often had short hospital stays (3 days or less, $51 \%$ vs $32 \%, p=0.001$ ) and were more often discharged during weekends/Mondays/holidays ( $33 \%$ vs $15 \%$, $\mathrm{p}=0.004)$.

Results from the regression analysis are shown in Table 2. In crude models, being (i) counselled during admission, (ii) contacted during the $1^{\text {st }}$ week after discharge, (iii) prescribed varenicline at discharge or during follow-up, and (iv) nurse-patient care continuity were each positively associated with 2-month smoking cessation. Adjusting for covariates, contact during the $1^{\text {st }}$ week after discharge and prescription of varenicline remained statistically significant. Entering all routines simultaneously into a single model, being prescribed varenicline was the only routine independently associated with smoking cessation at 2months (adjusted OR 4.09 [1.68-10.00], p=0.002).

Being a retrospective study, no harms, or unintended effects on account of the current study were noted or documented.

\section{Discussion}

The present study showed that implementing evidence-based, low cost- and readily available methods for smoking cessation can have a significant impact on smoking habits after an MI. Implementation resulted in a higher proportion of abstinent patients at 2-month follow up; $64 \%$ vs $54 \%$ during the reference period, and including only patients counselled during admission smoking abstinence was $73 \%$ vs $54 \%$.

To stop smoking following onset of CHD or after undergoing revascularization results in a considerably lower risk of death. Information and support for smoking cessation should be delivered routinely to all patients with a diagnosis of $\mathrm{CHD}^{3,9}$. This risk reduction is evident across strata by sex, age, index cardiac event, country, and year of study initiation ${ }^{3}$. 
According to SWEDEHEART data, being abstinent from smoking at 2-months after $\mathrm{MI}$ is associated with a $50 \%$ reduction in 10-year mortality risk (Figure 4$)^{6}$. The population of patients as well as the structure of follow-up in SWEDEHEART is practically equivalent to the present study's control period population. Hence, more abstinent patients 2-months after MI should be associated with a significant improvement regarding both long-term life expectancy.

At all six hospitals participating in the project, various forms of smoking cessation support were already in-place before project initiation. Thus, the implemented model was largely an implementation that strengthened and structured existing support. Focus was on assessing smoking status early, offering all present smokers brief verbal advice complemented with written material, and pharmacological treatment optimization. Implementation was performed without increasing personnel resources - and although it's difficult to estimate the exact cost for society - there is little doubt that a nurse-managed smoking cessation program after acute $\mathrm{Ml}$ is cost-effective. In a study by Quist-Paulsen, cost of a nurse-led smoking intervention program compared favourably to other treatment modalities for CHD patients, being approximately $1 / 25$ the cost of statins and angiotensin-converting enzyme inhibitors ${ }^{16}$. In another study by Krumholz et al that evaluated the cost-effectiveness of a similar nurse-led intervention, that included three hours of extra counselling time with a nurse, was cost-effective at an estimated $\$ 220 /$ year of life saved ${ }^{17}$. In the present study we estimate that the extra counselling time (patient identification, counselling during admission and more telephone contacts during follow-up) to be approximately 1-2 hours per patient. More time and resources for counselling in future studies and clinical praxis is most likely warranted, also since clinical studies show a positive relationship between number of counselling sessions and abstinence rate ${ }^{18,19}$. To achieve optimal effect, smoking cessation programmes should preferably include wide-ranging medical information, behavioral aspects, community-oriented methods and suitable pharmaco-therapeutic treatments ${ }^{8,18,20}$. Moreover, it has been proposed that telephone counseling and text messaging is effective for outpatients after a $\mathrm{Ml}^{21}$.

Out of all current smokers at baseline less than $10 \%$ were concomitantly using wet snuff during the reference and implementation periods, respectively. Use of wet snuff is common in Sweden, with $18 \%$ of men and $5 \%$ of women using wet snuff daily ${ }^{5}$ and using wet snuff as means to quit smoking cigarettes is quite common. The small number of current wet snuff users in our study, however, limited the possibilities for meaningful sub-analyses of this group.

As the CR nurses at the participating hospitals only work office hours Monday to Friday, lack of time to identify and subsequently counsel patients during admission was probably the major barrier to offering counselling during admission to more patients, but only $50 \%$ of the patients included in the current analysis received counselling. This in turn affected the CR nurses' possibility to improve treatment during admission and at discharge. Also, if the patients were not identified during admission, they were not contacted by telephone during the first week after discharge. Instead, first contact was at the initial CR assessment visit (2-3 weeks post discharge), as it was during the reference period and which is the general rule in Sweden. The importance of identifying patients early is supported in our data by the large 
difference in odds for smoking cessation when only including patients who received counselling (per protocol, OR 2.50 [1.42-4.41], $\mathrm{p}=0.002$ ) vs all patients treated during the implementation period (intention to treat, OR 1.60 [1.04-2.48], p=0.034). Given the potential benefits in gained lives and cost-effectiveness of a relatively simple intervention, increasing $\mathrm{CR}$ nurse resources to facilitate counselling even on weekends and during odd hours should be a priority.

Sweden has a low prevalence of daily smokers around 7\% yet the proportion of MI patients that smoke is $23 \%{ }^{5,6}$. Many European countries have significantly higher prevalence of smoking among MI patients, including Spain (41\%), Turkey (42\%), Serbia (46\%) and Cyprus (57\%) ${ }^{22}$. Accordingly, simple interventions such as the one evaluated in the current study, in countries with a high proportion of active smokers, could have major effects on the general prognosis post-MI. We encourage healthcare authorities to facilitate implementation of structured multi-component smoking cessation methods, which should be offered to every smoker who suffers an Ml.

The study has several strengths and limitation. The study had a relatively large sample size (383 patients) and several hospitals, both university hospitals and rural hospitals, were involved. All currently smoking MI patients admitted at the participating hospitals were included, increasing study representativeness. Missing data was also minimal. On the other hand, smoking status was assessed by self-report and no biochemical validation was performed to verify abstinence. Nevertheless, self-reporting is considered accurate in most smoking cessation studies ${ }^{23}$. The duration and intensity of smoking (i.e., $\mathrm{n}$ packages smoke per time unit, and years of smoking) is not available via SWEDEHEART or medical records. Some of the implemented routines were already in place at the participating hospitals before start of the project, also during the reference period. This likely reduced the difference between implementation and control periods. Although the association of each component of the new routines was assessed in the secondary analysis collinearity is plausible. The short follow-up of 2 months is a limitation. A longer follow-up of 6 or 12 months would have been preferred, as abstinence at 12 months is a good predictor for long-term abstinence ${ }^{24}$. However, as previously stated the Covid19 pandemic made a longer follow-up period unfeasible. Other factors than the implemented routines, such as public health interventions or general attitudes in society might have changed during the periods and influenced the results. However, major confounding effects of this sort seem unlikely since SWEDEHEART data has shown similar results regarding smoking cessation after $\mathrm{Ml}$ for ten years prior to and during the study period ${ }^{6}$.

In conclusion, our results from a real-life clinical setting show that implementing evidence-based and readily available methods for smoking cessation can have a significant positive impact on smoking cessation post-MI. The result was evident even though far from all patients were subject to all implemented routines. The most plausible barrier to implementing the new routines was lack of time for the CR nurses. Strengthening CR nurse resources for this important task should thus be a priority.

\section{Abbreviations}


BMI - Body mass index -

CHD - Coronary heart disease

$\mathrm{Cl}$ - Confidence interval

CR - Cardiac rehabilitation

EUROASPIRE - European Action on Secondary and Primary Prevention by Intervention to Reduce Events EVITA - Evaluation of Varenicline in - Smoking Cessation for Patients Post-Acute Coronary Syndrome -

Kg - Kilograms

LVEF - Left ventricular ejection fraction

MI - Myocardial infarction

NRT - Nicotine replacement therapy

OR - Odds ratio

StaRI - Standards for Reporting Implementation Studies -

STEMI - ST-elevation myocardial infarction -

SWEDEHEART - The Swedish Web-system for Enhancement and Development of Evidence-based care in Heart disease Evaluated According to Recommended Therapies

TnT - Troponin T

\section{Declarations}

\section{Author's contributions}

M.Le. and M.La. designed the study. M.Le., S.W., M.S., E.H. and M.Le. were involved in implementing the new routines at the participating hospitals. M.Le., S.W., H.Ö.M. and E.H. contributed to data collection. M.Le. and J.W .were responsible for data analysis. M.Le. and M.La. were responsible for drafting the manuscript. All authors reviewed the manuscript at several stages in the writing process.

\section{Additional information}

Competing interests' statement.

The authors have no competing financial or non-financial interests to declare. 
The study was funded by the Swedish Heart and Lung Association and Pfizer. The funding sources had no role in the design of the study or writing of the manuscript.

\section{References}

1. Teo, K. K. et al. Tobacco use and risk of myocardial infarction in 52 countries in the INTERHEART study: a case-control study. Lancet 368, 647-658, doi:10.1016/S0140-6736(06)69249-0 (2006).

2. Chow, C. K. et al. Association of diet, exercise, and smoking modification with risk of early cardiovascular events after acute coronary syndromes. Circulation 121, 750-758, doi:10.1161/CIRCULATIONAHA.109.891523 (2010).

3. Critchley, J. A. \& Capewell, S. Mortality risk reduction associated with smoking cessation in patients with coronary heart disease: a systematic review. JAMA 290, 86-97, doi:10.1001/jama.290.1.86 (2003).

4. United States Public Health Service Office of the Surgeon General. Smoking Cessation: A Report of the Surgeon General. Rockville, MD: Office of the Surgeon General, U.S. Department of Health and Human Services (2020).

5. Use of cigarettes, wet snuff and e-cigarettes (Swedish). Public Health Agency of Sweden. 2020. https://www.folkhalsomyndigheten.se/livsvillkor-levnadsvanor/andts/utveckling-inom-andtsanvandning-och-ohalsa/bruk/tobak-och-liknande-produkter/vuxnas-bruk-av-cigaretter-snus-och-ecigaretter/. Accessed 8 June 2021.

6. Vasko, P. et al. SWEDEHEART Annual report 2020. (Uppsala Clinical Research Center (UCR), Uppsala, Sweden, 2021).

7. Kotseva, K. et al. Lifestyle and impact on cardiovascular risk factor control in coronary patients across 27 countries: Results from the European Society of Cardiology ESC-EORP EUROASPIRE V registry. Eur J Prev Cardiol 26, 824-835, doi:10.1177/2047487318825350 (2019).

8. Ambrosetti, M. et al. Secondary prevention through comprehensive cardiovascular rehabilitation: From knowledge to implementation. 2020 update. A position paper from the Secondary Prevention and Rehabilitation Section of the European Association of Preventive Cardiology. Eur J Prev Cardiol, 2047487320913379, doi:10.1177/2047487320913379 (2020).

9. Piepoli, M. F. et al. 2016 European Guidelines on cardiovascular disease prevention in clinical practice: The Sixth Joint Task Force of the European Society of Cardiology and Other Societies on Cardiovascular Disease Prevention in Clinical Practice (constituted by representatives of 10 societies and by invited experts)Developed with the special contribution of the European Association for Cardiovascular Prevention \& Rehabilitation (EACPR). Eur Heart J 37, 2315-2381, doi:10.1093/eurheartj/ehw106 (2016).

10. Cahill, K., Stevens, S., Perera, R. \& Lancaster, T. Pharmacological interventions for smoking cessation: an overview and network meta-analysis. Cochrane Database Syst Rev, CD009329, doi:10.1002/14651858.CD009329.pub2 (2013). 
11. Eisenberg, M. J. et al. Varenicline for Smoking Cessation in Hospitalized Patients With Acute Coronary Syndrome. Circulation 133, 21-30, doi:10.1161/CIRCULATIONAHA.115.019634 (2016).

12. Sterling, L. H., Windle, S. B., Filion, K. B., Touma, L. \& Eisenberg, M. J. Varenicline and Adverse Cardiovascular Events: A Systematic Review and Meta-Analysis of Randomized Controlled Trials. $J$ Am Heart Assoc 5, doi:10.1161/JAHA.115.002849 (2016).

13. Windle, S. B. et al. Smoking abstinence 1 year after acute coronary syndrome: follow-up from a randomized controlled trial of varenicline in patients admitted to hospital. CMAJ 190, E347-E354, doi:10.1503/cmaj.170377 (2018).

14. Prugger, C. et al. Passive smoking and smoking cessation among patients with coronary heart disease across Europe: results from the EUROASPIRE III survey. Eur Heart J 35, 590-598, doi:10.1093/eurheartj/eht538 (2014).

15. Jernberg, T. et al. SWEDEHEART Annual report 2017. (Uppsala Clinical Research Center (UCR), Uppsala, 2018).

16. Quist-Paulsen, P., Lydersen, S., Bakke, P. S. \& Gallefoss, F. Cost effectiveness of a smoking cessation program in patients admitted for coronary heart disease. Eur J Cardiovasc Prev Rehabil 13, 274-280, doi:10.1097/01.hjr.0000192742.81231.91 (2006).

17. Krumholz, H. M., Cohen, B. J., Tsevat, J., Pasternak, R. C. \& Weinstein, M. C. Cost-effectiveness of a smoking cessation program after myocardial infarction. J Am Coll Cardiol 22, 1697-1702, doi:10.1016/0735-1097(93)90598-u (1993).

18. A clinical practice guideline for treating tobacco use and dependence: A US Public Health Service report. The Tobacco Use and Dependence Clinical Practice Guideline Panel, Staff, and Consortium Representatives. JAMA 283, 3244-3254 (2000).

19. Olenik, A. \& Mospan, C. M. Smoking cessation: Identifying readiness to quit and designing a plan. JAAPA 30, 13-19, doi:10.1097/01.JAA.0000520530.80388.2f (2017).

20. West, R., McNeill, A. \& Raw, M. Smoking cessation guidelines for health professionals: an update. Health Education Authority. Thorax 55, 987-999, doi:10.1136/thorax.55.12.987 (2000).

21. Kong, J. H. \& Ha, Y. [Effects of a smoking cessation program including telephone counseling and text messaging using stages of change for outpatients after a myocardial infarction]. J Korean Acad Nurs 43, 557-567, doi:10.4040/jkan.2013.43.4.557 (2013).

22. Snaterse, M. et al. Smoking cessation in European patients with coronary heart disease. Results from the EUROASPIRE IV survey: A registry from the European Society of Cardiology. Int J Cardio/ 258, 1-6, doi:10.1016/j.ijcard.2018.01.064 (2018).

23. SRNT Subcommittee on Biochemical Verification. Biochemical verification of tobacco use and cessation. Nicotine Tob Res 4, 149-159, doi:10.1080/14622200210123581 (2002).

24. Nohlert, E., Ohrvik, J., Tegelberg, A., Tillgren, P. \& Helgason, A. R. Long-term follow-up of a high- and a low-intensity smoking cessation intervention in a dental setting-a randomized trial. BMC Public Health 13, 592, doi:10.1186/1471-2458-13-592 (2013). 
Figures

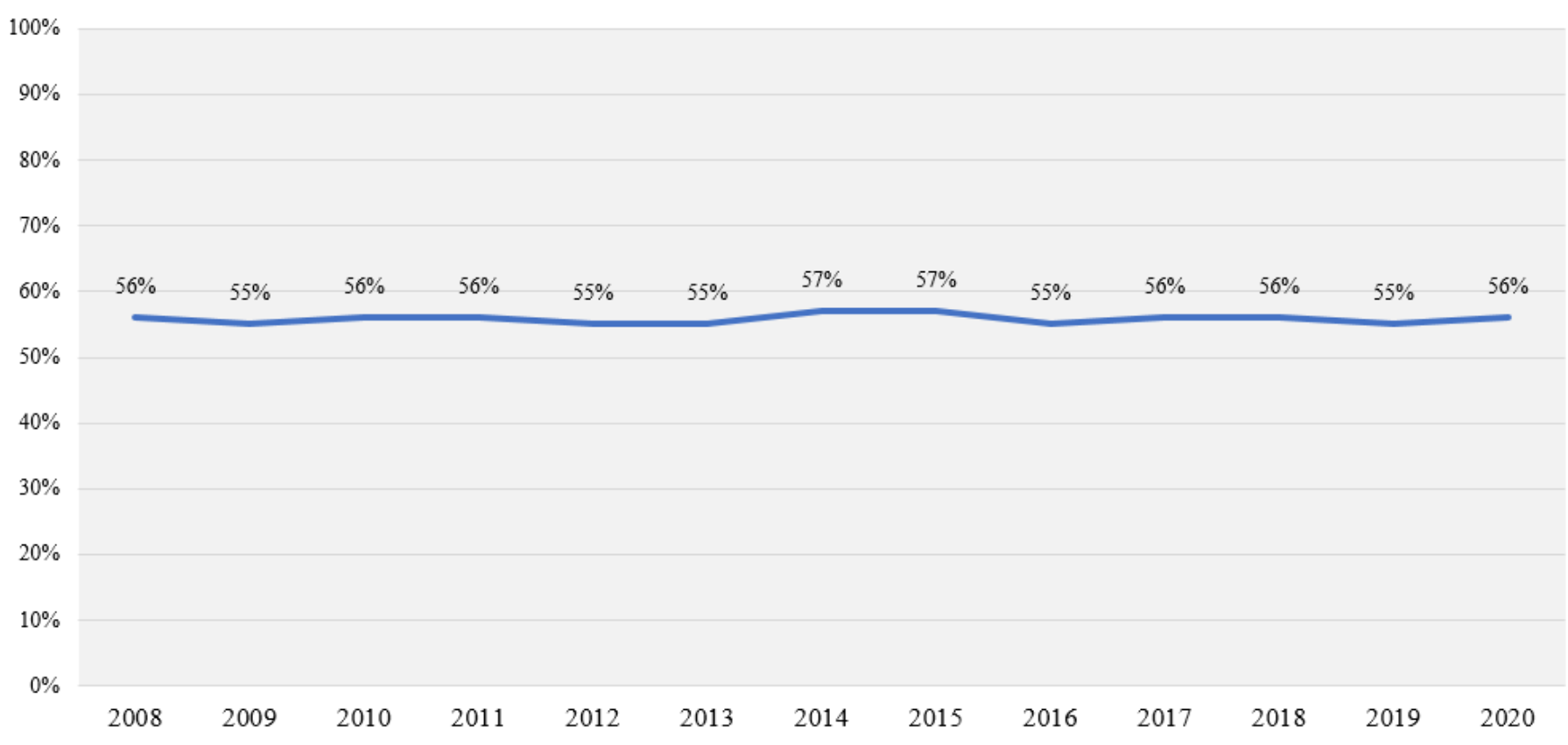

\section{Figure 1}

Annual trends in proportion of patients with Ml in Sweden, currently smoking at the time of the index event, who were abstinent at one-year follow-up (self-report). Adapted with permission from SWEDEHEART annual reports 2008-2020.

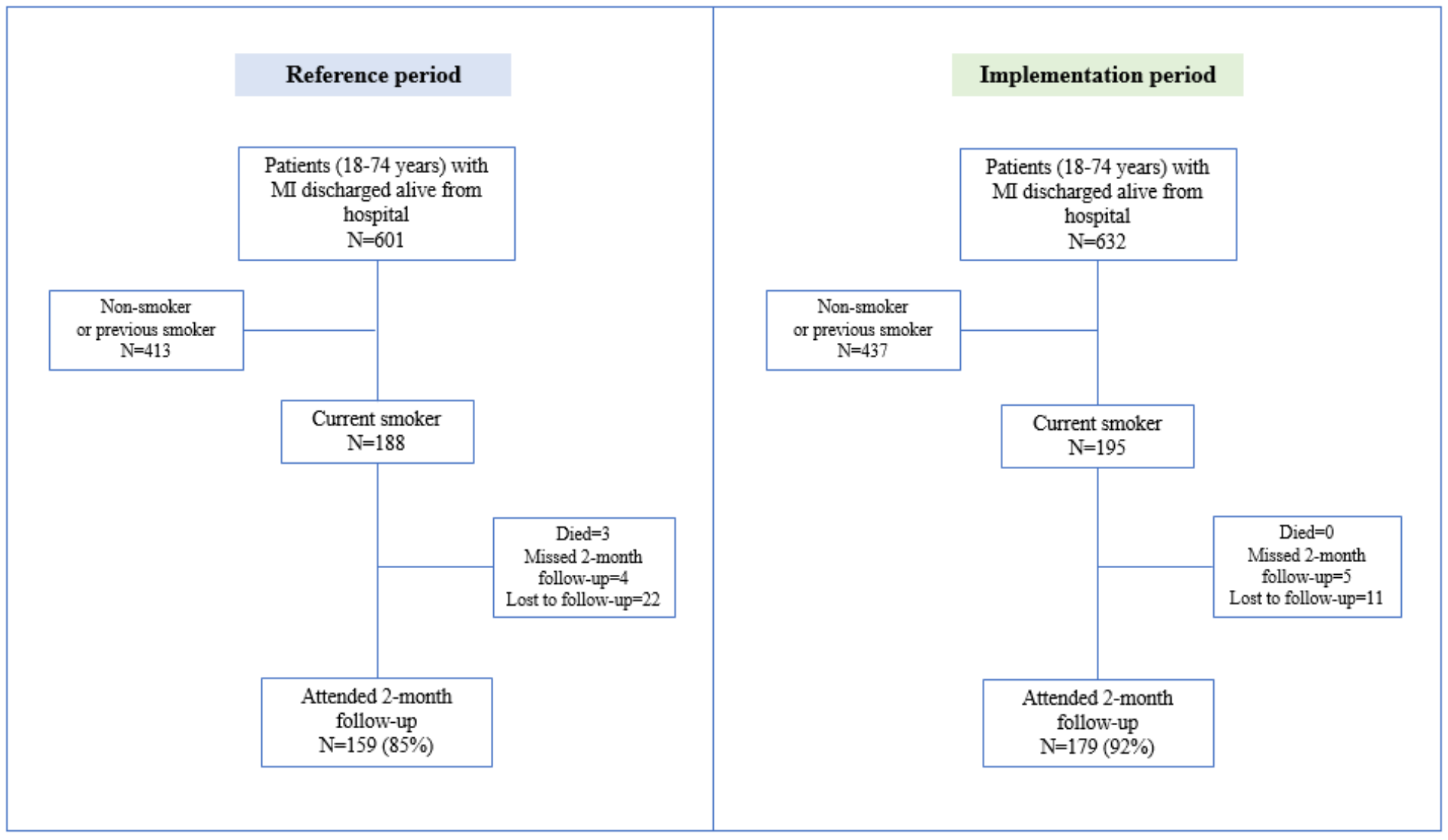


Figure 2

Flow chart for patients in the study. Ml, myocardial infarction.

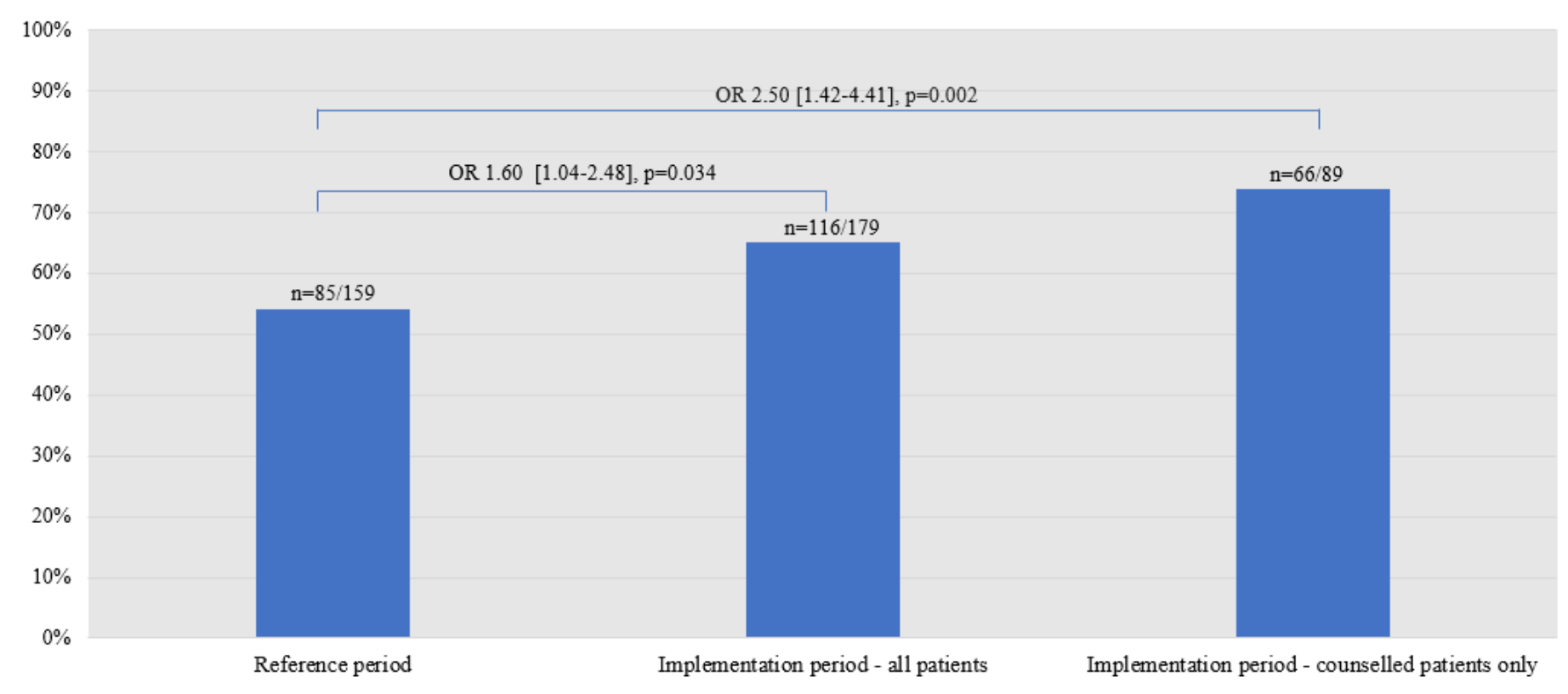

\section{Figure 3}

The proportion of abstinent patients and the crude odds of smoking abstinence at 2-month follow-up during the reference period (far left) and implementation period, for all patients (center) and for those counselled during admission only (far right). OR, odds ratio. 


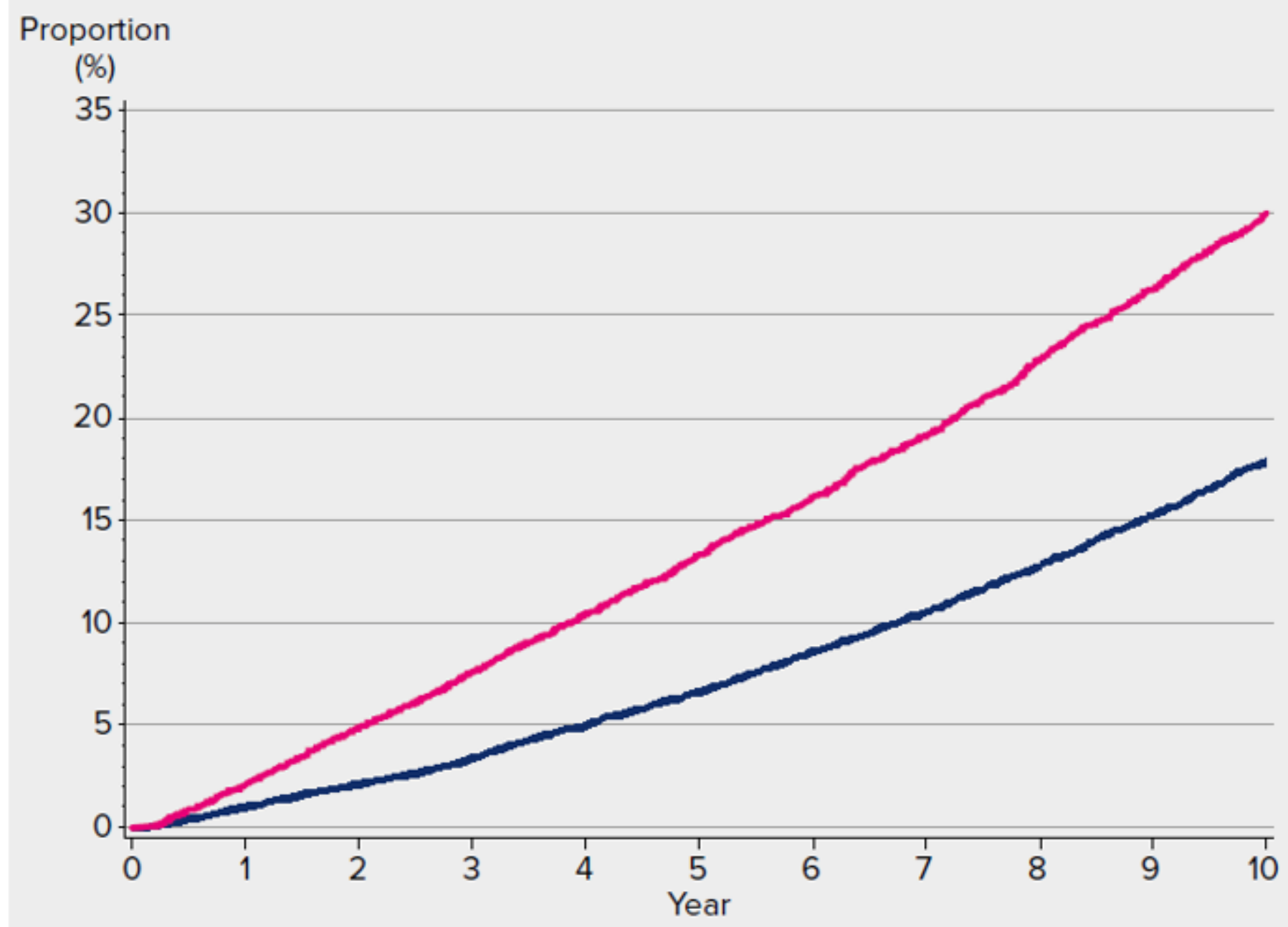

Abstinent at 2-month follow-up

Still smoking at 2-month follow-up

\section{Figure 4}

Crude mortality rate in patients who were smokers at the time of the Ml, relative to their smoking status at the 2-month follow-up. The graph includes all patients who attended their 2-month follow-up in 20052020 (approximately 100000 patients). Reprinted with permission from SWEDEHEART.

\section{Supplementary Files}

This is a list of supplementary files associated with this preprint. Click to download.

- SupplementaryDataset.pdf 